\title{
Un nuevo ensayo clínico con descontaminación digestiva selectiva
}

\section{A new clinical trial with selective digestive decontamination}

\author{
Paloma García-Hierro a, Miguel A. de la Cal ${ }^{b, *}$, Hendrick K.F. van Saene ${ }^{c}$ \\ y Luciano Silvestri ${ }^{\mathrm{d}}$
}

\author{
aServicio de Microbiología, Hospital Universitario de Getafe, Madrid, España \\ bervicio de Medicina Intensiva, Hospital Universitario de Getafe, Madrid, España \\ 'Department of Medical Microbiology, University of Liverpool, Liverpool, Reino Unido \\ ${ }^{\mathrm{d}}$ Department of Anaesthesia and Intensive Care, Presidio Ospedaliero, Gorizia, Italia
}

Recibido el 8 de abril de 2009; aceptado el 20 de abril de 2009

\section{Introducción}

De Smet et al ${ }^{1}$ han publicado un nuevo ensayo clínico sobre descontaminación digestiva selectiva (DDS). La DDS fue descrita en los años ochenta como una técnica para prevenir la infección respiratoria en los enfermos críticos ${ }^{2}$ y desde entonces se han realizado 57 ensayos clínicos con asignación aleatoria. Por lo tanto, es la técnica más extensamente evaluada en las unidades de cuidados intensivos (UCI). La mejor estimación de su efecto en la población general de enfermos críticos es que la administración del protocolo completo ${ }^{2}$ previene una neumonía por cada 5 enfermos tratados y una muerte por cada 21 enfermos tratados 3 . A pesar de estos efectos beneficiosos, no se ha generalizado el uso de DDS porque los expertos aducen, en contra de la evidencia existente, que: a) la reducción de la mortalidad es heterogénea en los diferentes ensayos clínicos, y b) puede conllevar un aumento de la resistencia antibiótica?.

De Smet et al presentan los resultados de un ensayo clínico, realizado en 13 centros de los Países Bajos, que incluye a 6.000 pacientes. Sus objetivos fueron comparar el im-

\footnotetext{
*Autor para correspondencia.

Correo electrónico: mcal@ucigetafe.com (M.A. de la Cal).
}

pacto en la mortalidad y la resistencia antibiótica de la aplicación del protocolo completo de DDS contra la descontaminación sólo orofaríngea -polimixina, tobramicina, anfotericina-y contra placebo.

\section{Diseño}

El método utilizado para realizar el ensayo clínico no es el convencional. Presenta diferencias relevantes. La asignación aleatoria se realiza por clusters. Es decir, en este caso el sujeto de la asignación aleatoria no es el enfermo, sino el orden en que cada unidad aplica un tipo de tratamiento durante 6 meses a todos los enfermos que cumplen los criterios de inclusión en el estudio. Este tipo de ensayos clínicos deben ser diseñados y analizados de modo diferente al de los ensayos clínico convencionales ${ }^{4}$. Las principales diferencias con otros ensayos clínicos están en el cálculo del tamaño muestral que debe corregirse al alza en los ensayos clínicos de cluster según el valor del efecto de diseño ${ }^{5}$ y en el análisis, que presenta mayor complejidad4. Personas poco conocedoras del método pueden considerar que el análisis estadístico realizado por De Smet et al es innecesario, pero, tal como refieren los autores, es imprescindible. Este diseño poco frecuente se justifica porque al recibir todos los enfermos el mismo tratamiento se previene el posible efecto cruzado (contaminación) entre los enfermos a 
quienes se da el tratamiento y a los que no se les da. Desde el primer ensayo clínico con asignación aleatoria que demostró que la DDS controla brotes de flora resistente ${ }^{6}$, se sabe que al tratar un grupo con DDS y erradicar a los portadores de este grupo, también disminuye el número de portadores del grupo tratado con placebo. La explicación más plausible es que la DDS disminuye la presión de colonización de la flora resistente al disminuir el número de portadores. El diseño propuesto, aun con sus dificultades de diseño y análisis, es el apropiado para evitar ese posible sesgo.

Otra fuente de sesgo potencial es la ausencia de ceguera (blinding) del estudio, cuya dirección más probable es hacia la hipótesis nula. Es decir, tendería a infravalorar la diferencia del efecto entre cada tratamiento. Los autores describen extensamente el control exhaustivo de sesgos, que incluye el análisis multivariable.

\section{Impacto en la mortalidad}

La odds ratio (OR) para los enfermos con DDS frente a los tratados con placebo es 0,835 (intervalo de confianza [IC] del $95 \%, 0,72-0,968)(p=0,016)$ y para los tratados con descontaminación orofaríngea, 0,858 (IC del 95\% 0,739-0,996) $(p=0,045)$. El número necesario para tratar de evitar una muerte es 28 y 34, respectivamente, para una mortalidad basal del $27,5 \%$

Los datos confirman que la reducción de la mortalidad de DDS frente a placebo es mayor y más precisa que la descontaminación orofaríngea (SDD, $p=0,016$; descontaminación orofaríngea, $p=0,045)$, aunque la diferencia entre ambas no alcance significación estadística.

Los valores encontrados en el estudio de De Smet et al no son estadísticamente diferentes de los previamente publicados. En el metaanálisis de referencia sobre $\mathrm{DDS}^{3}$ la OR para la mortalidad es 0,78 (IC del $95 \% 0,68-0,89)$ y el número necesario que hay que tratar para evitar una muerte es 21 (IC del 95\% 14-43), asumiendo un mortalidad basal del $29 \%$ Además, los resultados de los ensayos clínicos muestran homogéneamente esa reducción $\left(I^{2}=5 \%\right)$.

¿Cómo se explican estas diferencias? Posiblemente se deban a dos grupos de factores. En primer lugar, los relacionados con el diseño del estudio, ya comentados. En segundo lugar, los factores relacionados con la población estudiada. Frecuentemente, se ha intentado establecer la relación entre la reducción de la mortalidad y los diferentes factores de riesgo de la población estudiada. El factor más consistentemente relacionado es el score de gravedad al ingreso. En un estudio retrospectivo de los enfermos incluidos en 23 ensayos clínicos sobre DDS, Sun et al ${ }^{7}$ Ilamaron la atención sobre el hecho de que la reducción de la mortalidad sólo se producía en enfermos con una mortalidad esperada mayor del $20 \%$ La reducción de la mortalidad mostraba una relación lineal positiva: a mayor mortalidad esperada, mayor reducción de la mortalidad en los enfermos tratados con DDS. Este hallazgo ha sido confirmado en ensayos clínicos posteriores. Así Krueger et $\mathrm{al}^{8}$ no observaron una reducción significativa de la mortalidad en los enfermos tratados con DDS con un valor de APACHE II al ingreso <20. De J onge et al ${ }^{9}$ también apreciaron el mismo fenómeno: en los pacientes con un valor de APACHE Il en el percentil inferior no se observó ningún efecto de la DDS en la mortalidad. En el ensayo clínico holandés los enfer- mos con valor APACHE II $<20$ suponen el $54 \%$ En esa población no es esperable un descenso de mortalidad con ninguna intervención, salvo los cuidados generales. El impacto esperable del uso de DDS en esa población será en la reducción de la incidencia de neumonías, bacteriemias y, como luego veremos, en el control de la flora resistente.

Para estimar la relevancia clínica del efecto de la DDS en la mortalidad conviene compararlo con otras prácticas recomendadas en el tratamiento de los pacientes críticos. Así, por ejemplo, el riesgo relativo de mortalidad en los pacientes con síndrome de insuficiencia respiratoria aguda tratados con volumen corriente de $6 \mathrm{ml} / \mathrm{kg}$ frente a los tratados con $12 \mathrm{ml} / \mathrm{kg}$ es 0,78 (IC del 95\% 0,65-0,93), similar al de la DDS, en una población con una mortalidad basal del $40 \%$

\section{Impacto en las bacteriemias}

Los hallazgos de De Smet et al $^{1}$ confirman una reducción en la incidencia de bacteriemias por bacilos gramnegativos aerobios ( $B G N A)(O R=0,26$; IC del 95\% 0,17-0,38) similares a los encontrados en un metaanálisis de 51 ensayos clínicos $(O R=0,39$; IC del 95\%,0,24-0,63). Las bacteriemias por Candida spp. también descienden un $50 \%(O R=0,49$; IC del $95 \%, 0,21-1,11)^{10}$. Los antimicrobianos utilizados en la solución digestiva -polimixina, tobramicina, anfotericina-sólo tienen efecto en la flora gramnegativa y los hongos. Por lo tanto, no es de esperar una reducción en la flora grampositiva.

Otro hallazgo de interés es que una reducción de esa magnitud en la incidencia acumulada de bacteriemias sólo se observa cuando se aplica la DDS y no la descontaminación orofaríngea, lo que indica que las bacteriemias que se previenen son aquellas cuya flora tiene su origen en el aparato digestivo, que es el principal reservorio de Ios BGNA y de Candida spp.

\section{Impacto en la resistencia}

La estimación de la resistencia antibiótica en el ensayo clínico holandés se hace mediante estudios de prevalencia puntual sobre menos del $10 \%$ de la población estudiada. El método habitualmente recomendado para estimar la resistencia antibiótica es el cálculo de la incidencia de la flora resistente adquirida en la $\mathrm{UCl}$. Por ello, los datos aportados no son estrictamente comparables con los de otros estudios diseñados para conocer el impacto de la DDS en la resistencia antibiótica. No obstante, en el ensayo clínico de De Smet et al la administración de DDS conlleva una menor prevalencia de resistencia antibiótica de BGNA en comparación con el grupo placebo (descenso relativo del 50 al 75\% en muestras rectales). Este efecto no se observa con la administración de descontaminación orofaríngea.

La reducción de portadores de flora resistente confirma los hallazgos previamente descritos en un ensayo clínico, con una duración de 2 años, diseñado para valorar el efecto de la DDS en la resistencia11, en el que el riesgo relativo de adquirir BGNA resistentes a aminoglucósidos, cefalosporinas, carbapenemes o quinolonas se redujo un $40 \%$ (riesgo relativo $=0,61 ;$ IC del $95 \% 0,46-0,81$ ).

A pesar de estos resultados, los autores insisten en que "en las unidades con alto grado de endemia de bacterias 
gramnegativas multirresistentes o Staphylococcus resistente a meticilina, la DDS se asocia a un aumento en la selección de esos patógenos". Para apoyar esa afirmación se citan 2 ensayos clínicos ${ }^{12,13}$, obviando cualquier referencia a la ausencia de resistencia de Ios BGNA en 55 ensayos clíni$\cos ^{2,3}$ y en 6 estudios observacionales con uso de DDS durante al menos 2 años ${ }^{2}$.

El ensayo clínico de Verwaest et $\mathrm{al}^{13}$, que es el artículo reiteradamente referido por los oponentes a la DDS, debe ser considerado, por lo tanto, una excepción (outlier). Este ensayo expresa con claridad una de las limitaciones de la DDS y algunos errores a la hora de interpretar el efecto de la DDS. En primer lugar, los autores sólo proporcionan los datos de resistencia de los microorganismos hallados en las infecciones, cuando la valoración de la resistencia debe incluir también la de los portadores de flora resistente. En el grupo control, las infecciones causadas por enterobacterias resistentes fueron 36 en el grupo placebo y 25 en el grupo DDS, y las causadas por BGNA no fermentadores, 46 y 58, respectivamente. Las diferencias no fueron estadísticamente significativas. Este efecto inesperado -ausencia de reducción de las infecciones por BGNA resistentes-se puede justificar en parte porque esas infecciones no fueron precedidas por la colonización del aparato digestivo, que es donde es eficaz la DDS, ya que esta práctica previene la colonización y el sobrecrecimiento bacteriano en el intestino, lo que favorece la aparición de resistencia ${ }^{14,15}$.

También conviene llamar la atención sobre un error frecuente al administrar DDS. Descontaminar no es dar DDS, también requiere hacer cultivos de vigilancia de la flora orofaríngea y rectal para comprobar que se consigue el objetivo deseado: erradicar la flora potencialmente patógena del aparato digestivo. En el ensayo de Verwaerst et al ${ }^{13}$ el grupo de 200 pacientes tratados con DDS presentó 201 episodios de coIonización rectal. Al menos 61 (30\%) pacientes no recibieron una adecuada descontaminación. Es decir, un ensayo clínico que se cita como soporte para no recomendar la DDS presenta sesgos relevantes: no se estudia a los portadores, no se descontamina adecuadamente y, posiblemente, esa UCl tenía una alta incidencia de infecciones sin relación con un estado de portador previo, cuya prevención únicamente puede realizarse reforzando las medidas higiénicas y no con DDS.

La interpretación que hacen De Smet et al de los resultados del otro ensayo clínico citado sobre la resistencia ${ }^{12}$ no se ajusta a la información ni a la interpretación de los propios autores, que en un estudio posterior ${ }^{16}$, en el que comparan los datos de resistencia durante el ensayo clínico ${ }^{12}$ con el periodo anterior y posterior, concluyen literalmente: "durante un periodo de 2 años, ningún efecto en la resistencia antimicrobiana puede ser atribuido a la DDS".

En 6 de los 57 ensayos clínicos que evaluaron la eficacia de la $\mathrm{DDS}^{2}$, se ha comunicado el aumento de la incidencia de portadores de $\mathrm{S}$. aureus resistente a meticilina en unidades con endemia por ese microorganismo. Este hecho, recordado por De Smet el $\mathrm{al}^{1}$, es una limitación inherente a los antimicrobianos utilizados en la DDS (polimixina, tobramicina, anfotericina), sin cobertura frente a bacterias grampositivas. La adición de vancomicina a la pasta oral y a la solución digestiva ha demostrado ser eficaz y segura a largo plazo para erradicar $S$. aureus resistente a meticilina en unidades sin Enterococcus sp. resistente a vancomicina ${ }^{17-20}$.
Por lo tanto, la mejor evidencia disponible, incluidos los resultados de este ensayo clínico, no apoya la opinión de los autores, sino la opuesta: la DDS reduce el grado de resistencia antibiótica.

\section{Conclusiones}

El ensayo clínico de De Smet et $\mathrm{al}^{1}$ es el de mayor tamaño muestral publicado. Confirma la eficacia de la DDS en la mortalidad, con una posible subestimación vinculada al tipo de diseño y al elevado porcentaje ( $54 \%$ ) de enfermos con un índice de gravedad bajo. Es el primer ensayo clínico que muestra una reducción de la mortalidad estadísticamente significativa $(p=0,045)$ con la descontaminación orofaríngea. Asimismo, confirma que la DDS reduce la incidencia de bacteriemias y la adquisición de flora resistente. Parece aconsejable que los autores estimen el efecto ajustado de la DDS en la mortalidad en la población con mayor gravedad. Por último, se precisa un nuevo estudio multicéntrico en unidades con diferente incidencia de resistencia antibiótica adquirida para dar respuesta a los que insisten en que la DDS aumenta la resistencia antibiótica en contra de la evidencia actualmente disponible.

\section{Bibliografía}

1. De Smet AM, Kluytmans JA, Cooper BS, Mascini EM, Benus RF, Van der Werf TS, et al. Decontamination of the digestive tract and oropharynx in ICU patients. N Engl J Med. 2009;360:20-31.

2. Taylor N, Van Saene HKF, Abella A, Silverstri L, Vucic M, Peric M. Descontaminación digestiva selectiva. ¿Por qué no aplicamos la evidencia en la práctica clínica? Med Intensiva. 2007;31:136-45.

3. Liberati A, D’Amico R, Pifferi, Torri V, Brazzi L. Antibiotic prophylaxis to reduce respiratory tract infections and mortality in adults receiving intensive care. Cochrane Database Syst Rev. 2004; CD000022.

4. Campbell MK, Elbourne DR, Altman DG. CONSORT statement: extension to cluster randomised trials. BMJ . 2004;328:702-8.

5. Kerry SM, Bland J M. Sample size in cluster randomisation. BMJ . 1998; 316:549.

6. Brun-Buisson C, Legrand P, Rauss A, Richard C, Montravers F, Besbes $\mathrm{M}$, et al. Intestinal decontamination for control of nosocomial multiresistant gram-negative bacilli. Study of an outbreak in an intensive care unit. Ann Intern Med. 1989;110:873-81.

7. Sun X, Wagner DP, Knaus WA. Does selective decontamination of the digestive tract reduce mortality for severely ill patients? Crit Care Med. 1996;24:753-5.

8. Krueger WA, Lenhart FP, Neeser G, Ruckdeschel G, Schreckhase $\mathrm{H}$, Eissner $\mathrm{HJ}$, et al. Influence of combined intravenous and topical antibiotic prophylaxis on the incidence of infections, organ dysfunctions, and mortality in critically ill surgical patients: a prospective, stratified, randomized, double-blind, placebo-controlled clinical trial. Am J Respir Crit Care Med. 2002; 166: 1029-37.

9. De J onge $E$. Effects of selective decontamination of digestive tract on mortality and antibiotic resistance in the intensivecare unit. Curr Opin Crit Care. 2005;11:144-9.

10. Silvestri L, Van Saene HK, Milanese M, Gregori D, Gullo A. Selective decontamination of the digestive tract reduces bacterial bloodstream infection and mortality in critically ill patients. Systematic review of randomized, controlled trials. J Hosp Infect. 2007; 65: 187-203. 
11. De J onge E, Schultz MJ, Spanjaard L, Bossuyt PM, Vroom MB, Dankert J, et al. Effects of selective decontamination of digestive tract on mortality and acquisition of resistant bacteria in intensive care: a randomised controlled trial. Lancet. 2003;362:1011-6.

12. Hammond J M, Potgieter PD, Saunders GL, Forder AA. Doubleblind study of selective decontamination of the digestive tract in intensive care. Lancet. 1992;340:5-9.

13. Verwaest $C$, Verhaegen J, Ferdinande P, Schetz M, Van den BG, Verbist $L$, et al. Randomized, controlled trial of selective digestive decontamination in 600 mechanically ventilated patients in a multidisciplinary intensive care unit. Crit Care Med. 1997;25:63-71.

14. Salyers AA, Moon K, Schlesinger D. The human intestinal tract - a hotbed of resistance gene transfer? Part I. Clinical Microbiology Newsletter. 2007;29:17-22.

15. Van Saene HK, Taylor N, Damjanovic V, Sarginson RE. Microbial gut overgrowth guarantees increased spontaneous mutation leading to polyclonality and antibiotic resistance in the criticaIly ill. Curr Drug Targets. 2008; 9:419-21.

16. Hammond J M, Potgieter PD. Long-term effects of selective decontamination on antimicrobial resistance. Crit Care Med. 1995; 23:637-45.
17. Cerda E, Abella A, De la Cal MA, Lorente JA, Garcia-Hierro P, Van Saene HK, et al. Enteral vancomycin controls methicillinresistant Staphylococcus aureus endemicity in an intensive care burn unit: a 9-year prospective study. Ann Surg. 2007;245: 397-407.

18. De la Cal MA, Cerda E, Van Saene HK, Garcia-Hierro P, Negro E, Parra ML, et al. Effectiveness and safety of enteral vancomycin to control endemicity of methicillin-resistant Staphylococcus aureus in a medical/ surgical intensive care unit. J Hosp Infect. 2004; 56: 175-83.

19. Silvestri L, Van Saene HK, Milanese M, Fontana F, Gregori D, Oblach $L$, et al. Prevention of MRSA pneumonia by oral vancomycin decontamination: a randomised trial. Eur Respir J. 2004; 23:921-6.

20. Viviani M, Van Saene HK, Dezzoni R, Silvestri L, Di LR, Berlot G, et al. Control of imported and acquired methicillin-resistant Staphylococcus aureus (MRSA) in mechanically ventilated patients: a dose-response study of enteral vancomycin to reduce absolute carriage and infection. Anaesth Intensive Care. 2005; 33:361-72. 